\title{
A Proposed Astronomy Learning Progression For Remote Telescope Observation
}

Timothy F. Slater, University of Wyoming, USA

Andrea C. Burrows, University of Wyoming, USA

Debbie A. French, University of Wyoming, USA

Richard A. Sanchez, University of Wyoming, USA

Coty B. Tatge, University of Wyoming, USA

\begin{abstract}
Providing meaningful telescope observing experiences for students who are deeply urban or distantly rural place-bound-or even daylight time-bound-has consistently presented a formidable challenge for astronomy educators. For nearly 2 decades, the Internet has promised unfettered access for large numbers of students to conduct remote telescope observing, but it has only been in recent years that the technology has become readily available. Now that this once fanciful possibility is becoming a reality, astronomy education researchers need a guiding theory on which to develop learning experiences. As one departure point, we propose a potential learning progression anchored on one end with recognizing that stars visible at night have describable locations and predictable motions, and anchored at the other with distant robotic telescopes can be programmed to record specific astronomical data for later analysis.
\end{abstract}

Keywords: Science Education; Astronomy; Discipline-Based Education Research; Learning Progressions

\section{INTRODUCTION}

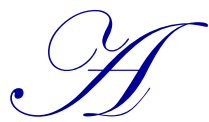

stronomy is about wondering what is in the sky high above Earth's surface. At its core, astronomy is an observational science and provides rich opportunities for systematic observing, describing, cataloging, predicting, comparing, contrasting and studying the light from faraway places. In contrast to the hierarchical steps in the traditionally taught scientific method, astronomy is a science rarely done with control groups or hypothesis testing. Instead, the task of astronomy is to find clever ways to gather the tiniest amount of light data about astronomical objects, typically with telescopes looking up into the inky skies.

If observing is indeed the essence of astronomy, then a telescope would be a vital tool to the astronomer. In the same way, it seems that for the astronomy educator, an accessible and easy-to-use telescope would be a vital learning tool for the astronomy student. It is a common experience that people talking casually about astronomy readily share their stories of vivid memories about the first time a planet came into view, such as the ringed-planet Saturn, through a telescope. We won't belabor further the importance of telescope observing experiences as it has been argued eloquently elsewhere (Beare, 2007; Camacho, Holt, Marley, \& Roberts, 2009; Culver \& Craine, 2000; Hoban, Keating, Sachs, Laughlin, \& Yesha, 2002; Oswalt, 2003; Privon et al., 2009; Smith, 2008).

If we agree that telescopes are the primary tool of astronomy, we wonder why many students learning astronomy have had few - if any - opportunities to look through telescopes and marvel at the revealed wonders of the night sky. Certainly, not every school has telescopes in their storeroom or employs a teacher who personally owns one that he or she is able to share with students. Although slightly more affordable this decade than in previous ones, telescopes are generally expensive. Inexpensive ones do exist, but a significant challenge with inexpensive telescopes is that their supporting mounts and tripods are often difficult for novices to use, too quickly resulting in overwhelming frustration for the user. 
When schools do have access to telescopes, there are several complications when using them. First, school happens during the day, and telescopes are most often used at night. Telescope observing is best done under dark skies away from light, and most schools in the US are located within cities filled with light pollution. Additionally, most campuses are well lit for safety and to prevent vandalism, making school yard campus grounds often difficult to use because of outdoor lighting. Second, the reality of personnel is challenging. Getting parents to provide transportation to and from school at night is problematic. Finding enough people with expertise using the telescopes to work with student groups is demanding, as is finding experts to share their expertise of astronomy. Third, even when all of these other issues are circumvented, inclement weather also manages to intercede. No matter how large, telescopes are not able to look through substantive layers of clouds. No location is completely immune. For some types of telescope observing, even seemingly clear skies can be disappointing because there is too much turbulence in the upper atmosphere for images to be stable enough to view satisfactorily. Some celestial objects are only visible at certain times of the year or from geographic locations on the Earth's other hemisphere or within the darkness of a crescent moon phase. Finally, Borders and colleagues (2010) reported that misconceptions plague astronomy students, especially regarding scale and distance, which can affect data collection and analysis. Most of these issues do have some clever workarounds, but by and large they are significant challenges of using telescopes. This article describes new opportunities for doing astronomical observing and proposes a possible learning progression for building effective learning environments.

\section{OPTIONS FOR ACCESSING TELESCOPES}

In response to the challenges previously discussed, some teachers have reframed their astronomy courses to think of observing in terms of student engagement and awareness of their environment. Generations of teachers have asked their students to chart the shape and position of the moon each night for a month, measure the changing length of noontime shadows, or carefully monitor the direction of the setting sun across the western horizon, all without a telescope (Slater, 1995). Such telescope-free approaches to observing are long standing practices across the community of experienced astronomy teachers.

Still, telescope-free approaches are unsatisfying to some teachers. One recent solution to the high cost of telescopes was developed during the United Nations-sponsored International Year of Astronomy in 2009. Galileo's worldview altering telescope observations of the Universe happened approximately 400 years ago. His iconic observations included observing moons orbiting the planet Jupiter, monitoring the phases of Venus, finding craters on the Moon, and discovering spots on the Sun. How can students experience observations and collect data similar to Galileo's?

To help students across the globe be able to make meaningful observations, a team of astronomers and engineers produced a high quality, low cost telescope known widely as the GalileoScope (GalileoScope, 2014). This refracting telescope has a diameter of about 2 inches and was mass-produced to cost, amazingly, under \$25 USD. These telescopes are constructed by the user and are highly durable (Pompea \& Isbell, 2009). Lesson plans exist online that can be used to help students assembling their GalileoScopes to learn conceptual principles of optics. As such, the GalileoScope project has successfully made low-cost telescopes available and affordable to many school classrooms. 


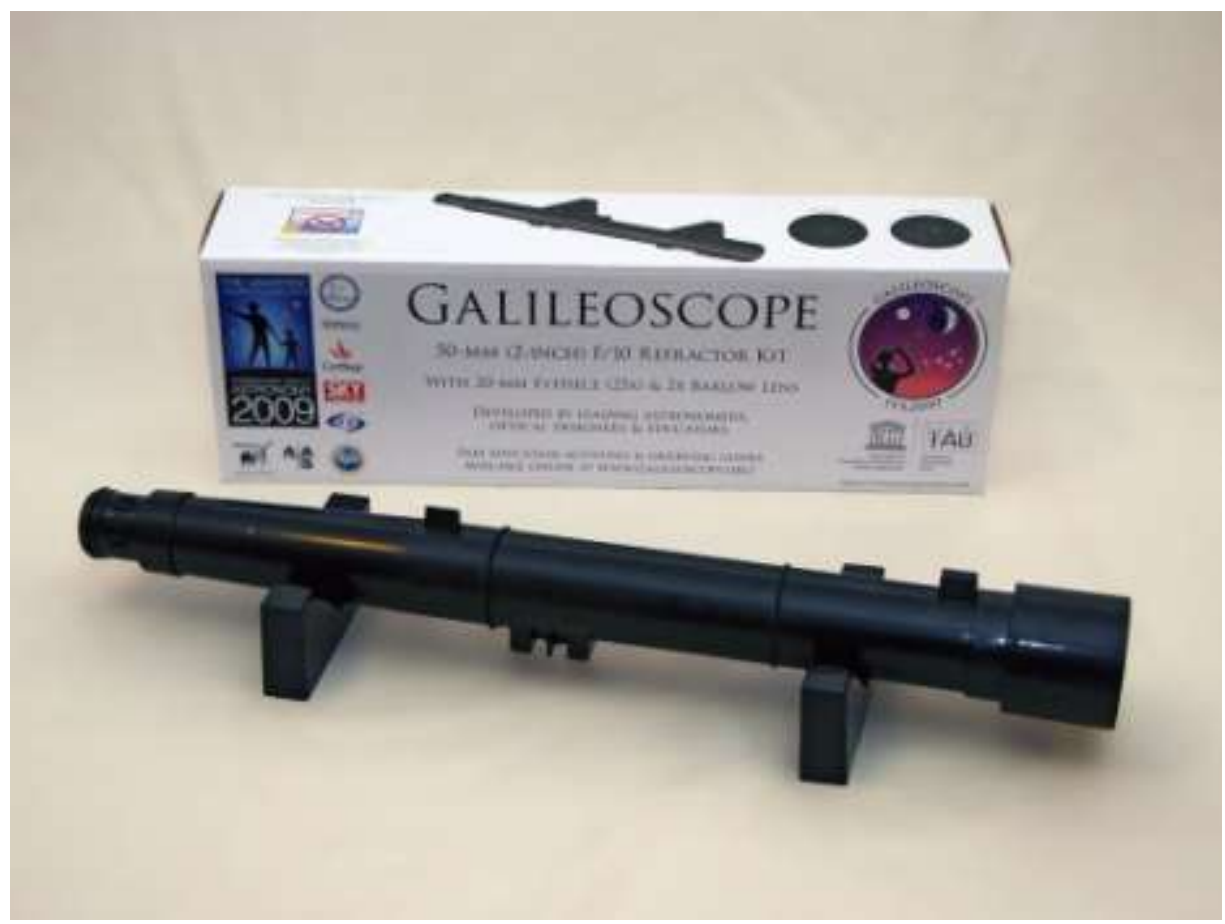

Figure 1 - Galileoscope

GalileoScope from GalileoScope.org, Licensed under CC-PD

http://www.astronomy2009.org/static/archives/images/screen/galileoscopewithbox.jpg

Perhaps surprising to some, most communities around the US have groups of dedicated amateur astronomers who have made considerable investments in telescope purchases. These amateur astronomy clubs are frequently willing to bring their telescopes to a school campus or, more commonly, will issue invitations to school children to attend their observing gatherings - called star parties - at nearby dark sites. The National Aeronautics and Space Administration (NASA) and the Astronomical Society of the Pacific have created a national network of amateur astronomy club members who will allow students to look through their personally owned telescopes (Manning, 2008). Many amateur astronomy clubs have a long history of collaborating with schools to provide students with telescope observing opportunities at low to no cost.

\section{REMOTELY AND ROBOTICALLY CONTROLLED INTERNET TELESCOPES}

One way to get around telescope barriers is to harness the power of the Internet to control a telescope remotely. Remote observing is where a telescope user is actually moving and controlling a telescope in a far-away place using an Internet connection in real time. "Electronic access to computer-controlled telescopes equipped with digital cameras can solve some of these difficulties by enabling students and their teachers to access internetcontrollable telescopes, and consult more readily with experts" (Stencel, Harland, Hannahoe, Bisque, \& Rice, 2002, p. 719). In this sense, students obtain astronomical data from a telescope far from where they are physically located.

For nearly 2 decades, the Internet has promised unfettered access for large numbers of students to conduct remote telescope observing. During that time, there have been a few highly successful projects (including, among others, Danaia \& McKinnon, 2007; Gershun, Slater \& Berryhill, 2014; Lacy, 2008; Mayo, Schweitzer, Clark, Hoban, \& Melsheimer, 2001; McKinnon\& Mainwaring, 2000; Pennypacker \& Miller, 2009; Stencel, Harland, Hannahoe, Bisque, \& Rice, 2002), but most of these programs have necessarily had to limit the total number of students who could access these telescopes. Fortunately, in the last few years, remote and robotic telescope technology and Internet access have become more readily available so that larger numbers of students could obtain easy and low-cost access. Among those that can potentially handle large numbers of students available at the time of this writing, Slooh, iTelescope, and MicroObservatory have active programs, described below, and it is anticipated that Los Cumbres Global Telescope Network is soon to become widely available. There are undoubtedly 
others soon to be available, but these provide some sense of the near-term possibilities. In the end, the goal is probably best summarized by international astronomy educator Robert Hollow (2000, p. 162):“. . . the use of the internet and remote access telescopes allow students to undertake challenging research and make worthwhile contributions to professional programs."

Although a number now exist, the most highly subscribed remote telescope is known as Slooh (pronounced "slew"), which is a homophone word that astronomers also use to describe swinging a telescope from one target to another (Slooh, 2014). For a nominal subscription fee, Slooh observers look through an Internet camera viewed live through a web browser with Adobe Flash Player plug-in. It was not the first remote telescope, but it was the first that offered "live" viewing through a telescope via the web. Students can find this exhilarating! Slooh telescopes are located in Spain, Chile, and Australia. Images are downloaded immediately, as opposed to a delay when they are emailed later, which has been the conventional method until recently. Students can download images (see Figure 2) instantaneously to their computer or smart cell phone as well as post their images automatically to social media sites (e.g., Facebook). These images are in a commonly editable .png image format that can be readily modified in ubiquitous software packages similar to MS Word and MS PowerPoint. Early classroom implementation evidence points to success using this simple interface with students across a wide range of ages (Gershun, Berryhill, \& Slater, 2012).

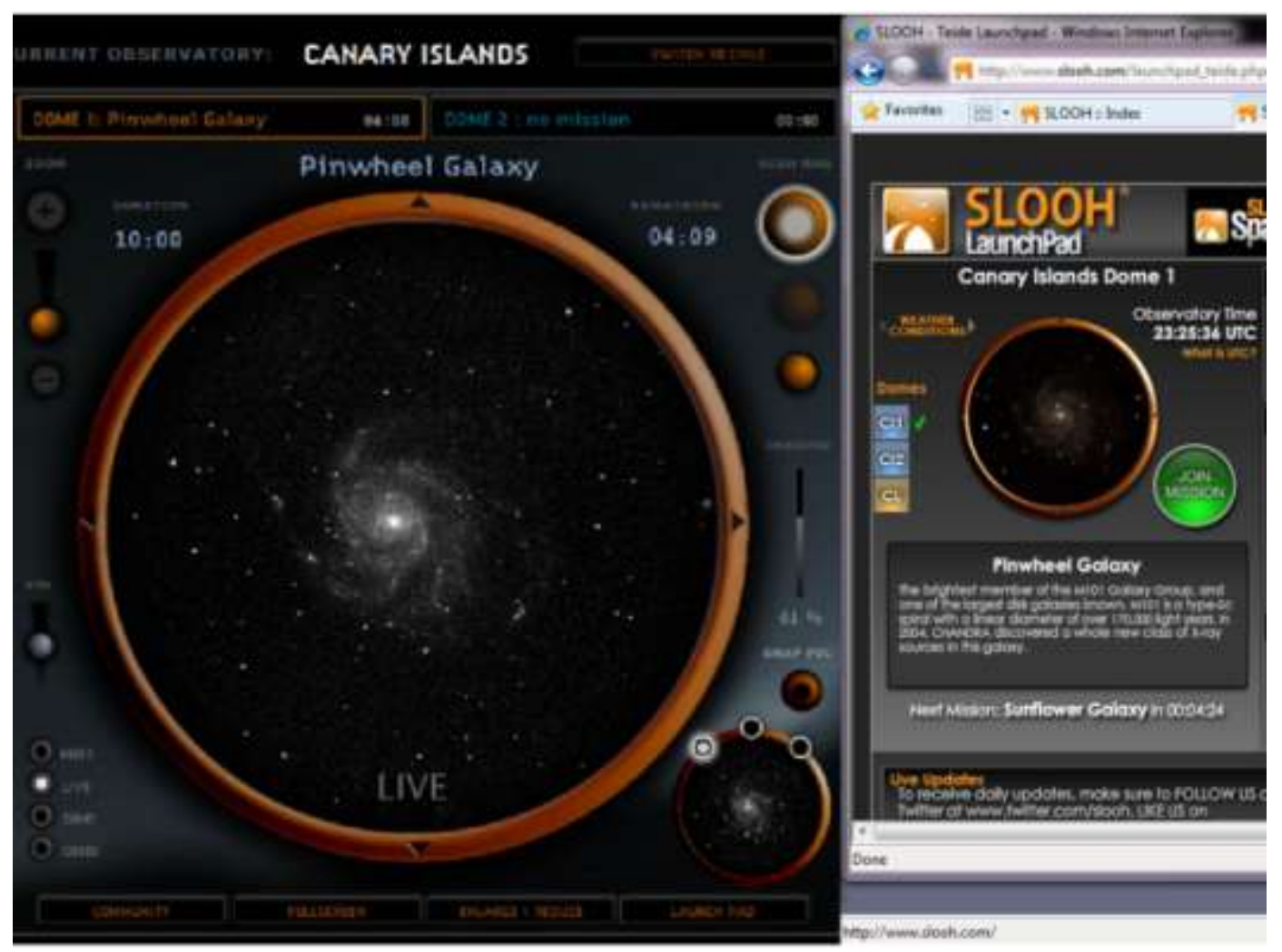

Figure 2 - Slooh.Com Observing Interface,

Image Created By The Author With Permission From Slooh.Com

Another currently active, cost-effective remote telescope is the itelescope.com program. Unlike Slooh it does not provide real-time, live viewing experiences; however, itelescope.com does give students the opportunity to obtain astronomy data in real time across a wide variety of celestial objects through various filters. Also, accompanying the website are two instructional modules: one module for Stellarium - a free digital interactive sky chart for personal computers - and one for its launchpad observing interface. Itelescope provides access to approximately 20 telescopes available across the world, including one observatory in Australia, one in Spain, and two in the United States. These remote telescopes make it possible for users to plan multiple observing runs ahead 
of time. This tool would be useful to allow students to finish all observation planning and reserving within the allotted class time, without requiring them to allocate extra time outside of the classroom because it would run automatically. All of the data would then be saved in an online database in multiple image formats.

An alternative to real-time, Internet-based, remotely-controlled telescopes, robotically-controlled telescopes are also available to students. This is the type of research telescope that most professional astronomers use today, and it is programmed during the daytime to observe a series of objects for precise amounts of time (Querci \& Querci, 2000). Then a robotic telescope runs nearly autonomously during the night. Such a process allows a telescope to be used most efficiently and effectively by carefully predetermining which targets to view optimally over the course of a night. This results in one telescope observing more objects in a single night than when controlled in real time, and many more users can benefit from the telescope. Another name for this type of telescope is a batch-queued telescope, and most professional telescopes being built today are of this type.

Students can use a number of these robotically-controlled telescopes around the world. Probably the easiest and cheapest of the robotically-controlled telescopes for students to access and use is the system of microobservatory telescopes positioned around the US known as OWN, which stands for Observing With NASA (2014). During the day, students request the OWN telescopes to take images of the night sky with an easy-to-use point and click interface (see Figure 3). The system makes recommendations to users about length of exposure and which filters might be useful. Then, the next day after a clear night in which the telescope operates, the users are emailed a copy of their image and given a URL to download their image (and images others have requested) in several image formats, including those professional astronomers use. The OWN telescope site provides users with software and short tutorials on how to process and extensively edit the obtained images. OWN has been available to an evergrowing number of students and its positive impact on student learning has been extensively described elsewhere (Gould, Dussault, \& Sadler, 2001; Sadler et al., 2007).

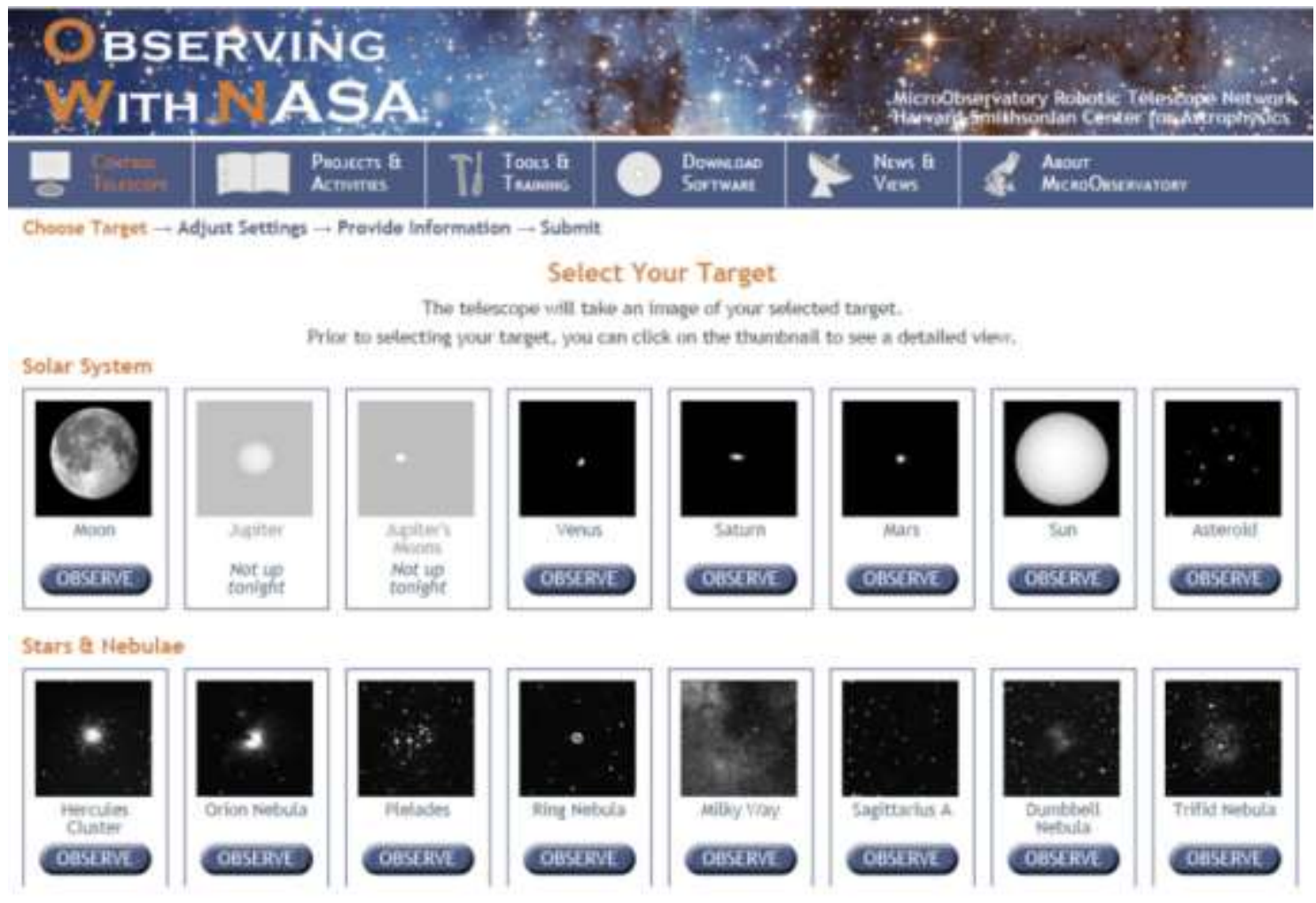

Figure 3 - MicroObservatory.org Observing Interface http://mo-www.harvard.edu/cgi-bin/OWN/Own.pl 
Bigger and more comprehensive telescope network options are on the near horizon. For example, the Las Cumbres Observatory Global Telescope Network is planning on deploying 1-meter and 0.4-meter robotic telescopes for educational use in addition to their existing 2-meter telescopes in Hawaii and Australia (LCOGT, 2014). Science education researchers, working teachers, and classrooms engaged with the LCOGT telescope networks are showing strong conceptual and attitudinal gains in science and technological understanding (Danaia, McKinnon, Parker, Fitzgerald, \& Stenning, 2012). Another solution, in addition to data available from remote and robotic telescopes, is many of the world's observatories and space probes have placed much of their scientific data online, easily accessible from the Internet. Some of these have adopted citizen science approaches to providing their data to the public, many of which have easily implementable classroom solutions (Slater, Slater, \& Lyons, 2010).

Unquestionably, remote and robotic observing — broadly defined—have great potential to overcome many of the barriers of using telescopes in the classroom as well as promoting student projects. Programs such as the Global Network of Astronomical Telescopes (GNAT) and Wide Field Infrared Survey Explorer (WISE), if carefully utilized, have the potential to be able to solve the rapidly growing large-enrollment class problems. And most promisingly, looking toward the future where many more schools are moving their astronomy classes to online learning environments rather than face-to-face classes, remote and robotic observing in one form or another will be the only viable solution. None of this solves the desire to have students get outside and let a million- or billion-yearold photon impact your eye, but it is a step toward increasing the number of students who might have a chance to actually do astronomy.

These resources provide a rich opportunity to integrate science, technology, engineering, and mathematics (STEM) across a course. While we generally think about using Internet telescopes in the college introductory ASTRO 101 course, we have no reason to suspect that this would not also be appropriate for a secondary school course. As an example, a model program implemented in Chicago Public Schools in conjunction with the University of Chicago Internet Project connected students to astronomy and STEM fields using the citizen-science project Galaxy Zoo and the Sloan Digital Sky Survey astronomical database. By incorporating more observing with robotic and remote telescopes, Bhat (2013) reported that the instructional team was able to increase the level of STEM integration dramatically.

\section{PROPOSED ASTRONOMY LEARNING PROGRESSION FOR REMOTE TELESCOPE OBSERVATION}

Once remote and robotic telescope observation opportunities are widely and equitably available to students learning astronomy, the astronomy teaching community inherits the arduous task of developing meaningful learning environments and effective instructional sequences. We are certain that a build [the telescope] and they will learn approach will be fleetingly attractive, but wholly ineffective based on both the common sense of experience and the science education literature (Slater, Slater, \& Bailey, 2010, pp. 17-28).

In recent years, the broader science education community has adopted the use of "learning progressions" to guide the development of instructional sequences (Duschl, Maeng, \& Sezen, 2011). The concept of learning progressions can be briefly described as the sequence of cognitive growth steps a learner encounters when deeply and flexibly learning a concept. At one end of the sequence, called the lower anchor, is the entering-level, conceptual understanding that a novice learner holds. At the upper end of the sequence, the exiting-level of conceptual understanding is known as the upper anchor, and is the desired ending point of instruction. The seminal touchstone learning progression is best characterized by the decade-long work done on by Smith and Wiser (2006) on the atomic-molecular theory.

Learning progressions have been purportedly used to guide the National Research Council's Framework for K-12 Science Education (Quinn, Schweingruber, \& Keller, 2012) and have been influencing some work in astronomy education (Plummer, 2012; Plummer \& Krajcik, 2010). A surprisingly large body of literature surrounding learning progressions has been exhaustively explained and justified elsewhere by prominent science educators including Alonzo and Gotwals (2012) as well as Duschl et al. (2011), and a detailed discussion is beyond the scope of this article. 
It is our position that the conceptual framework of learning progressions has great potential to guide both a new astronomy education research agenda and eventually the development of effective learning experiences. In that light, we propose the following tentative learning progression for remote telescope observation as a first step in preparing for the imminent and equitable availability of remote and robotic telescope systems.

Proposed Remote Telescope Observation Learning Progression

1. Small dots of light in the night sky, called stars, are visible each clear night [lower anchor]

2. Stars have names and positions in the sky that can be described and located on sky maps

3. Stars change their position in the sky over the course of a night and over the year

4. Most stars look like infinitely small dots through a telescope pointed at a prescribed location

5. Some star-like objects are not stars, but other objects like planets, and appear different in a telescope

6. Some nearby objects are visible in a telescope are not visible to the naked eye, such as Jupiter's moons, Saturn's rings, craters on the moon, phases of Venus

7. Some distant objects visible in a telescope are not visible to the naked eye, such as galaxies, nebulae, and even other stars

8. Data from a telescope can be gathered through hand-drawn sketches, digital photographs, and other less familiar scientific instruments and take the form of an image or set of numbers

9. Color-images are created by combining and overlaying multiple images in different wavelengths

10. A computer can be programmed to point a telescope and gather data for the observer in real-time at nearby or faraway locations

11. A computer can be programmed to point a telescope and gather data for the observer at a faraway location, including in outer space, even when the observer is not nearby or even awake

12. Computer-controlled telescopes fill online databases that can be accessed over the Internet for analysis

13. Detailed analysis of astronomical objects can be accomplished by assigning a data collection task to a robotically controlled telescope or by querying a database of previous observations [upper anchor]

Figure 4 - Proposed Remote Telescope Observation Learning Progression

In this first-steps proposed learning progression for remote telescope observation, the lower anchor is set for what we predict is the incoming-level of cognitive understanding for a complete novice: Small dots of light in the night sky, called stars, are visible each clear night. This is based on our collective experiences in teaching thousands of students across a wide range of ages. At the upper end, we propose that the upper anchor be set at such a position where a student would have sufficiently deep and flexible understanding of using telescopes remotely and robotically that they would be well-positioned to ask a scientifically researchable question and design a reasonable strategy to pursue evidence to conduct this study: Detailed analysis of astronomical objects can be accomplished by assigning a data collection task to a robotically controlled telescope or by querying a database of previous observations. Between the two is what we anticipate to be a measurable and predictable cognitive pathway of conceptual understandings that hint at learning experiences that may move students from one level to another. As a community of astronomy education researchers, we do know that the pathways from misunderstanding to understanding are often unexpected, as illustrated by Sadler (1998). Unquestionably, whereas the lower anchor is based upon a cognitive-level of understanding, the upper anchor is influenced by what we as astronomy education researchers desire learners to achieve. In this way, we would expect that different teams of astronomy education researchers might conclude a different upper anchor for this progression, even given the same consensus lower anchor starting point. We suspect that this is a common weakness in the broader concept of learning progressions overall.

Upon consideration, some scholars might view a learning progression as nothing more than a simple "rational task analysis" like those described by Baker, Corbett, and Koedinger (2007). In a similar way, other scholars might question how learning progressions are substantively different from works such as the American Association for the Advancement of Science (AAAS, 2001) Atlas of Scientific Literacy or other online conceptual concept maps described by Sumner and colleagues (2005). In essence, we do not disagree that these are highly similar, if not conceptually the same, as the learning progressions. What we do predict is that if the scholarly astronomy teaching community does not quickly develop a literature describing deeper understandings of how students learn to observe, our community will have thrust upon us well-intentioned, but largely ineffective, online telescope interfaces and corresponding curricular products created without any guidance. It is to this end that we propose this learning progression with the intent that discipline-based researchers will develop an agenda to determine how astronomy students best learn to use, and come to value, telescope observing as part of their astronomy learning experience. 


\section{AUTHOR INFORMATION}

Drs. Slater and Burrows are professors in the Secondary Education Department at the University of Wyoming. Co-authors French, Sanchez, and Tatge are Ph.D. students in the College of Education at the University of Wyoming who, along with their professors, are studying how best to improve astronomy learning using integrated STEM teaching strategies. Their work influences the development of astronomy curriculum and integrated STEM education for students across the K-12 and early college years spectra.

\section{ACKNOWLEDGEMENTS}

This material is based in part upon work supported by the US National Science Foundation under Grant Numbers 1108238 and 1211112 and the Wyoming Excellence in Higher Education Endowment. Any opinions, findings, and conclusions or recommendations expressed in this material are those of the author(s) and do not necessarily reflect the views of the National Science Foundation or any specific funding entity.

\section{REFERENCES}

1. American Association for the Advancement of Science. Atlas of science literacy. Washington, DC: Author.

2. Alonzo, A. C., \& Gotwals, A. W. (2012). Learning progressions in science: Current challenges and future directions. New, NY: Springer.

3. Baker, R. S., Corbett, A. T., \& Koedinger, K. R. (2007). The difficulty factors approach to the design of lessons in intelligent tutor curricula. International Journal of Artificial Intelligence in Education, 17(4), 341-369.

4. Bhat, S. (2013, September 5). Astronomy course builds STEM foundation for Chicago students, teachers. UChicago News. Retrieved from http://news.uchicago.edu/article/2013/09/05/astronomy-course-buildsstem-foundation-chicago-students-teachers

5. Beare, R. (2007). Investigation into the potential of investigative projects involving powerful robotic telescopes to inspire interest in science. International Journal of Science Education, 29(3), 279-306.

6. Borders, K., et al. (2010, July). Infrared astronomy and education: Linking infrared whole sky mapping with teacher and student research. $38^{\text {th }}$ COSPAR Scientific Assembly, p. 17.

7. Camacho, A., Holt, R., Marley, S., \& Roberts, S. (2009). Robotic telescopes - Classroom activities at the cutting edge of science. Space Science Reviews International Journal, 90, 73-82.

8. Culver, R., \& Craine, E. (2000). A Global Network of Astronomical Telescopes (GNAT) node as a tool for student research projects. American Astronomical Society $197^{\text {th }}$ AAS Meeting, \#87.29. Bulletin of the American Astronomical Society, 32, 1560.

9. Danaia, L., \& McKinnon, D. H. (2007). Common alternative astronomical conceptions encountered in junior secondary science classes: Why is this so? Astronomy Education Review, 6(2), 32-53.

10. Danaia. L., McKinnon, D.H., Parker, Q., Fitzgerald, M., \& Stenning, P. (2012). Space to grow: LCOGT.net and improving science engagement in schools. Astronomy Education Review, 11(1), 1-14.

11. Duschl, R., Maeng, S., \& Sezen, A. (2011). Learning progressions and teaching sequences: A review and analysis. Studies in Science Education, 47(2), 123-182.

12. GalileoScope (2014). Found at URL: http://galileoscope.org.

13. Gershun, D., Berryhill, K., \& Slater, T. F. (2012, December). Evaluating SLOOH robotic telescopes for formal educational use. Poster presented at the American Geophysical Union Fall Conference, San Francisco, CA.

14. Gershun, D., Slater, T. F., \& Berryhill, K. (2014). Mixed-methods study that examines science teachers' perceptions of SLOOH robotic telescope for teaching astronomy. Latin-American Journal of Astronomy Education, 17, 7-37.

15. Gould, R., Dussault, M., \& Sadler, P. M. (2007). What's educational about online telescopes? Evaluating 10 years of MicroObservatory. Astronomy Education Review, 5(2), 127-145.

16. Hoban, S., Keating, J., Sachs, J., Laughlin, D., \& Yesha, Y. (2002, January). Science investigation system for telescopes in education research. Proceeding of the $36^{\text {th }}$ Hawaii International Conference on System Sciences. (pp. 7-pp). IEEE Computer Society. 
17. Hollow, R. P. (2000). The student as scientist: Secondary student research projects in astronomy. Astronomical Society of Australia, 17, 162-167.

18. Lacy, C. (2008). Teaching with web-based telescopes. American Astronomical Society, AAAS Meeting \#212, \#38.10. Bulletin of the American Astronomical Society, 40, 238.

19. LCOGT (2014). URL: http://www.lcogt.net

20. Manning, J., Gurton, S., Gibbs, M., Zevin, D., Berendsen, M., \& Fraknoi, A. (2008). Intermediary astronomy: Education through the leveraging of networks, partnerships and intermediaries at the Astronomical Society of the Pacific. Communicating Astronomy With the Public, 1, 310.

21. Mayo, L., Schweitzer, A., Clark, G., Hoban, S., \& Melsheimer, T. (2001). Global TIE (Telescopes in Education). American Astronomical Society, $199^{\text {th }}$ AAS Meeting, \#22.03; Bulletin of American Astronomical Society, 33, 1341.

22. McKinnon, D. H., \& Mainwaring, A. (2000). The Charles Sturt University remote telescope project: Astronomy for primary school students. Publications of the Astronomical Society of Australia, 17(02), 125128.

23. Observing With NASA (2014). URL http://mo-www.harvard.edu/OWN

24. Oswalt, T. (2003). The future of small telescopes in the new millennium. Dordrecht, Germany: Kluwer Academic Publishers.

25. Pennypacker, C., \& Miller, P. (2009, Month). International Astronomical Search Collaboration: An online student-based discovery program in astronomy. Paper presented at the American Geophysical Union Fall Meeting, San Francisco, CA. Abstract \#ED34A-05.

26. Plummer, J. D. (2012). Challenges in defining and validating an astronomy learning progression. In Learning progressions in science (pp. 77-100), Alonzo, A. C., \& Gotwals, A. W. Editors, Rotterdam, The Netherlands: SensePublishers.

27. Plummer, J. D., \& Krajcik, J. (2010). Building a learning progression for celestial motion: Elementary levels from an earth-based perspective. Journal of Research in Science Teaching, 47(7), 768-787.

28. Pompea, S. M., \& Isbell, D. (2009). The International Year of Astronomy 2009: New approaches and novel resources for physics classrooms. The Physics Teacher, 47(7), 428-433.

29. Privon, G., Beaton, R., Whelan, D., Yang, A., Johnson, K., \& Condon, J. (2009). The importance of handson experience with telescopes for students. E-print archive. Instrumentation and Methods for Astrophysics. Retrieved from arXiv preprint arXiv:0903.3447

30. Sadler, P. M., Gould, R., Leiker, P. S., Antonucci, P., Kimberk, R., Deutsch, F., \& Hoffman, B. (2001). MicroObservatory net: A network of automated remote telescopes dedicated to educational use. Journal of Science Education and Technology, 10(1), 39-55.

31. Sadler, P. M. (1998). Psychometric models of student conceptions in science: Reconciling qualitative studies and distractor-driven assessment instruments. Journal of Research in Science Teaching, 35(3), 265296.

32. Slater, S. J., Slater, T. F., \& Bailey, J. M. (2010). Discipline-Based Education Research: A Scientist's Guide (pp. 17-28). New York, New York: WH Freeman.

33. Slater, S. J., Slater, T. F. \& Lyons, D. J. (2011). Teaching scientific inquiry with GalaxyZoo. The Physics Teacher, 49(2), 94-96.

34. Slater, T. F. (2007). Using remote-control telescopes in ASTR 101. In A. Fraknoi (Ed.), Cosmos in the classroom symposium on teaching introductory astronomy. San, Francisco, CA: Astronomical Society of the Pacific.

35. Slater, T. F. (1995). AstroNotes: Teaching the science of Moon gazing. The Physics Teacher, 33, 186-187.

36. Slooh (2014). URL: http://www.slooh.com

37. Smith, C. L., Wiser, M., Anderson, C. W., \& Krajcik, J. (2006). Implications of research on children's learning for standards and assessment: A proposed learning progression for matter and the atomicmolecular theory. Measurement: Interdisciplinary Research \& Perspective, 4(1-2), 1-98.

38. Smith, T. C. (2008, May). Remote Observing and Beyond: Student Research and Analysis Programs at the Dark Ridge Observatory. In Society for Astronomical Sciences Annual Symposium (Vol. 27, p. 131).

39. Stencel, R., Harland, H., Hannahoe, R., Bisque, S., \& Rice, M. (2002). The Student Telescope Network (STN) experiment. American Astronomical Society, 200 ${ }^{\text {th }}$ AAS Meeting, \#47.05. Bulletin of the American Astronomical Society, 34, 719. 
40. Sumner et al. (2005). Linking learning goals and educational resources through interactive concept map visualizations. International Journal on Digital Libraries, 5(1), 18-24.

41. Querci, F. R., \& Querci, M. (2000). Robotic telescopes and networks: New tools for education and science. Astrophysics and Space Science, 273(1-4), 257-272.

42. Quinn, H., Schweingruber, H., \& Keller, T. (Eds.). (2012). A framework for K-12 science education: Practices, crosscutting concepts, and core ideas. Washington, DC:National Academies Press. 